\title{
One-Pot Synthesis and Characterization of Platinum-Coated Magnetite Nanoparticles for Magnetosensitive Catalyst
}

\author{
Quanguo $\mathrm{He}^{*}$, Zhaohui Wu, Lei Zeng and Chunyan Huang \\ Green Packaging and Biological Nanotechnology Laboratory, Hunan University of Technology, \\ Zhuzhou 412008, People's Republic of China
}

(Received March 18, 2011; accepted June 10, 2011)

Key words: platinum-coating, magnetosensitive catalyst, magnetite nanoparticle, nanocomposites, catalysis

Recent catalyst advances in nanoparticle-based-core synthesis have enabled the precise control of the size, shape, and composition of noble metal nanoparticles, enabling their extended catalytic activity and outside acting force responsiveness. Here, we report the design of a magnetosensitive catalyst model that consists of magnetite nanoparticles with a Pt metal coating shell $\left(\mathrm{Fe}_{3} \mathrm{O}_{4} @ \mathrm{Pt} \mathrm{NPs}\right)$ in a one-pot-synthesis method. The fabrication of the nanocomposite catalyst was achieved by one-stage reduction of chloroplatinic precursor ions. Monodisperse Pt-coated nanoparticles with a size of $c a$. $50 \mathrm{~nm}$ have been characterized by transmission electron microscopy, high-resolution transmission electron microscopy, energy-dispersive spectroscopy, X-ray photoelectron spectroscopy, ultraviolet-visible spectroscopy and superconducting quantum interference device magnetometry. The $\mathrm{Fe}_{3} \mathrm{O}_{4} @ \mathrm{Pt}$ NPs obtained under appropriate conditions without any surfactant possess a saturation magnetization of about $27.3 \mathrm{emu} \mathrm{g}^{-1}$ and demonstrate a good magnetic response that endows them with potential magnetosensitive catalysis application. Furthermore, the design concept used in the $\mathrm{Fe}_{3} \mathrm{O}_{4} @ \mathrm{Pt}$ NPs magnetosensitive catalyst can be extended to other metal/metal oxide compositions.

\section{Introduction}

Monodisperse magnetic composite nanoparticles (NPs) are of interest for both technological and fundametal reasons. From an applied standpoint, nanoscale architectures of composite NPs have attracted tremendous interest because of their potential applications in microelectronics, ${ }^{(1)}$ catalysis, ${ }^{(2)}$ photocatalysis, ${ }^{(3)}$ magnetic

"Corresponding author: e-mail: hequanguo@126.com 
devices, ${ }^{(4)}$ chemisorptions, ${ }^{(5)}$ aerosols, ${ }^{(6)}$ and powder metallurgies. ${ }^{(7)}$ From a basic research perspective, magnetic NPs have attracted extensive interest because of their unique magnetic properties. Such composites can be tailored with desired physical properties for further applications. Additionally, the promise of nanoparticle application is broadened further by the introduction of the core-shell system, for instance, having a magnetite $\left(\mathrm{Fe}_{3} \mathrm{O}_{4}\right)$ core encapsulated in a protective and perhaps even biocompatible shell. ${ }^{(8)}$

Meanwhile, the preparation, characterization and application of metal nanoparticles have been the focus of significant research effort over the past decade. ${ }^{(9-11)}$ The properties of the metal of interest change considerably at nanoscale dimensions owing to differences in both physical and chemical properties. ${ }^{(12-15)}$ These changes in properties lead to interesting applications in the field of catalysis, ${ }^{(8,14,15)}$ energy storage devices, ${ }^{(16)}$ optronics, ${ }^{(17)}$ magnetic devices, ${ }^{(18)}$ and biosensors. ${ }^{(19)}$ The high surface area-to-volume ratios that nanoparticles exhibit facilitate the effective use of expensive metals in catalysis. ${ }^{(11)}$ Therefore, the creation and development of nanosized materials with catalysis metal shells have brought important and promising techniques into the field of catalyst applications. Although much research has been carried out, there is still the paramount challenge for the wide application of noble metal nanoparticles as catalysts in the catalyst industry, i.e., how to separate and recycle them completely from the products. Thus, it is necessary to develop new catalysts with the inner magnetic cores and outer shell consisting of the catalytic species for ease of separation by external magnetic field application.

Thus far, the reports on the magnetic nanoparticles as catalyst supports or carriers are mainly focused on the magnetic iron oxide NPs coated with silica, carbon, gold, silver, polymer, etc. ${ }^{(20-27)}$ There are few reports on the synthesis of platinum-coated magnetite NPs. Platinum has been widely used for catalysis; however, platinum is a noble metal with limited resource availability and a high price. How to realize the effective manipulation, recovery and reutilization of it is currently still a major challenge for platinum-like noble metal catalysis. Given this point, employing a magnetite core and a platinum-coated shell would be an effective candidate solution. Moreover, they can be prepared by methods that range from mechanical to gas-phase to chemical. As a competitive alternative to magnetic NP preparation, the sonochemical method has drawn our attention owing to its ability to fabricate novel materials with unusual properties. The chemical effects of ultrasound arising from acoustic cavitation are beneficial to form a new phase, and have a shear effect on agglomeration, which is prone to prepare highly monodispersive nanoparticles. This method has been applied to the synthesis of various nanocomposites such as Fe-Co alloy NPs, ${ }^{(28)}$ core-shell-type $\mathrm{ZnO}$ nanorod and $\mathrm{CdS}$ NPs, ${ }^{(29)}$ and air-stable gold-coated magnetite NPs, ${ }^{(30)}$ and its versatility has been successfully demonstrated in many composite NP preparations.

Herein, we report a one-pot-synthesis method of monodisperse Pt-coated magnetite composite NPs $\left(\mathrm{Fe}_{3} \mathrm{O}_{4} / \mathrm{Pt} \mathrm{NPs}\right)$ without surfactants by sonolysis of the solution of $\mathrm{HPtCl}_{6} \cdot 6 \mathrm{H}_{2} \mathrm{O}$ and 3-aminopropyltriethoxysilane (APTES)-coated magnetite NPs with further reduction of hydrazine hydrate. Moreover, the reaction mechanism is also discussed briefly. 


\section{Experimental Procedure}

\subsection{Chemicals and reagents}

Ferric chloride $\left(\mathrm{FeCl}_{3} \cdot 6 \mathrm{H}_{2} \mathrm{O}, 99.0 \%\right)$ was purchased from Tianjin Bodi Chemicals Co., Ltd., ferrous chloride $\left(\mathrm{FeCl}_{2} \cdot 4 \mathrm{H}_{2} \mathrm{O}, 99.0 \%\right)$ from Tianjin Shuangchuan Chemicals Co., Ltd., 3-APTES from Sigma, and sodium hydroxide $(\mathrm{NaOH})$ from Hunan Huihong Chemicals Co., Ltd. Chloroplatinic acid $\left(\mathrm{H}_{2} \mathrm{PtCl}_{6} \cdot 6 \mathrm{H}_{2} \mathrm{O}\right)$ was purchased from Shanghai Zhongyuan Chemicals Co. Ethanol $\left(\mathrm{CH}_{3} \mathrm{CH}_{2} \mathrm{OH}, 95 \%\right)$ was purchased from Hunan Huihong Chemicals Co. Hydrazine hydrate $\left(\mathrm{N}_{2} \mathrm{H}_{5} \mathrm{OH}\right)$ was purchased from Shanghai Zhongyuan Chemicals Co. All the chemicals were of analytical grade and were used directly. Water $\left(T=25^{\circ} \mathrm{C}, 18.2 \mathrm{M} \Omega\right)$ was purified using SUPER ATER-II water purification systems, and this ultrapure water was used throughout the experiment. The $\mathrm{NdFeB}$ magnet, purchased from a local market, was used to separate magnetic particles in the washing and selection steps.

\subsection{Characterization}

Ultraviolet-visible spectroscopy (UV-Vis) spectra were acquired with a Purkinje General T-1901 spectrophotometer. The spectra were collected over the range of 200 $700 \mathrm{~nm}$.

Transmission electron microscopy (TEM) was performed using a Hitachi-600 Electron Microscope $(20 \mathrm{kV})$. The nanoparticle samples dispersed in ethanol solution were cast onto a carbon-coated copper grid sample holder, followed by evaporation at room temperature.

High-resolution transmission electron microscopy (HRTEM) images were observed on a JEOL JEM-2010 (FET) transmission electron microscope at an acceleration voltage of $200 \mathrm{kV}$, and the samples were dissolved in methanol and dropped on copper grids.

X-ray photoelectron spectroscopy (XPS) measurements were made using a Kratos Axis Ultra DLD. This system uses a focused monochromatic Al X-ray $(1,486.6 \mathrm{eV})$ source for excitation and a spherical section analyzer. The percentages of individual elements detected were determined from the relative composition analysis of the peak areas of the bands. The relative peak areas and their corresponding sensitivity factors were used to provide relative compositions.

Scanning electron microscopy (SEM) with energy dispersive spectroscopy (EDS) studies were carried out using a Hitachi $3000-\mathrm{N}$ microscope operated at $25 \mathrm{kV}$. Samples for SEM were prepared by deposition of the particles onto an Al substrate by vacuum drying the nanoparticles in air.

Superconducting quantum interference device (SQUID) magnetometry measurements were performed using a SQUID magnetometer (Quantum Design MPMS XL-7). Magnetic susceptibility $M-H$ curves were obtained at $T=300 \mathrm{~K}$ and $5 \mathrm{~K}$, where $M$ is the magnetization and $H$ is the applied magnetic field, which were measured as a function of temperature in a magnetic field.

\subsection{Experiment process}

The magnetite NPs and relevant APTES coating were described in detail elsewhere; thus, the APTES-coated $\mathrm{Fe}_{3} \mathrm{O}_{4}$ NPs were prepared accordingly. ${ }^{(30)}$ Then, at $80 \mathrm{kHz}$ (the 
ultrasonic frequency), $15 \mathrm{ml}$ of the APTES-coated $\mathrm{Fe}_{3} \mathrm{O}_{4} \mathrm{NPs}$ and $10 \mathrm{ml}$ of the $\mathrm{H}_{2} \mathrm{PtCl}_{6}$ solution $(0.1 \mathrm{~mol} / \mathrm{L})$ were mixed, and $16 \mathrm{ml}$ of hydrazine hydrate was then dropped into the mixture solution until the color changed from yellow to black. The resultant dark material was precipitated and separated by a magnetic field and centrifugation. The precipitated product was washed with ethanol and redispersed in ethanol $\left(\mathrm{Fe}_{3} \mathrm{O}_{4} / \mathrm{Pt} \mathrm{NPs}\right.$ sample).

\section{Results and Discussion}

\subsection{Proposed mechanism}

The main feature of the reaction mechanism is that the ultrasonic reduction reaction proceeds via the aqueous solution rather than in the solid state, and thus, the metal particles are formed by nucleation and growth from solution. As illustrated in Fig. 1, the synthesis of the $\mathrm{Fe}_{3} \mathrm{O}_{4} / \mathrm{Pt}$ nanocomposite includes three steps: first, we synthesized colloidal solutions of $\mathrm{Fe}_{3} \mathrm{O}_{4} \mathrm{NPs}$ by coprecipitation (step 1). The mechanism of preparing the APTES-functionalized $\mathrm{Fe}_{3} \mathrm{O}_{4}$ involves the hydroxyl groups on the $\mathrm{Fe}_{3} \mathrm{O}_{4}$ surface reacting with the ethoxy groups of the APTES molecules, leading to the formation of $\mathrm{Si}-\mathrm{O}$ bonds (step 2) and leaving the terminal amino $\left(-\mathrm{NH}_{2}\right)$ groups available for the immobilization of Pt (step 3). The APTES-coated $\mathrm{Fe}_{3} \mathrm{O}_{4}$ NPs show strong chelating

Step 1: $\quad \mathrm{Fe}^{2+}+2 \mathrm{Fe}^{3+}+8 \mathrm{OH}^{-} \rightarrow \mathrm{O} \downarrow+4 \mathrm{H}_{2} \mathrm{O}$

Step 2:

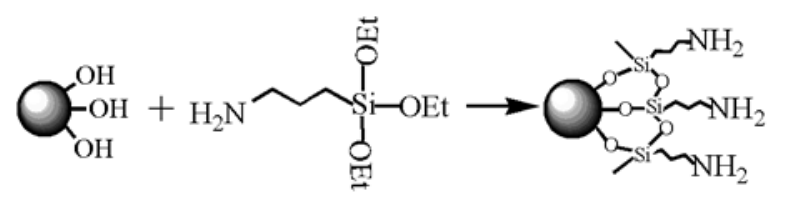

$\left(\mathrm{O}^{\mathrm{R}}\left(\mathrm{NH}_{2}\right)_{\mathrm{x}}\right)$

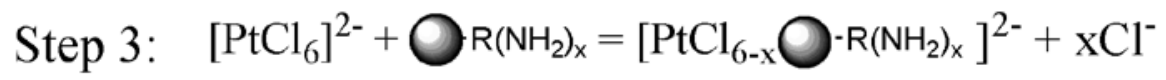
$x=1,2,3,4$

$\left[\mathrm{PtCl}_{6-\mathrm{x}} \mathrm{O}\left(\mathrm{NH}_{2}\right)_{\mathrm{x}}\right]^{2-}+\mathrm{N}_{2} \mathrm{H}_{5} \mathrm{OH} \rightarrow \mathrm{O} \downarrow+\mathrm{HCl}+\mathrm{N}_{2} \uparrow+\mathrm{H}_{2} \mathrm{O}$ $\mathrm{Fe}_{3} \mathrm{O}_{4} / \mathrm{Pt} \mathrm{NPs}$

$\mathrm{H}_{2} \mathrm{PtCl}_{6}+\mathrm{N}_{2} \mathrm{H}_{5} \mathrm{OH}=\mathrm{Q} \downarrow+6 \mathrm{HCl}+\mathrm{N}_{2} \uparrow+\mathrm{H}_{2} \mathrm{O}$ Pt NPs

Fig. 1. Synthetic scheme for the preparation of the Pt-coated magnetite NPs. 
ability for Pt (IV) ions $\left(\left[\mathrm{PtCl}_{6}\right]^{2-}\right)$ by the long pair of terminal amino groups, and the interactions can be further enhanced by mutually attractive electrostatic interactions when the two components are oppositely charged (step 2, " $\mathrm{R}$ " refers to the $\mathrm{Fe}_{3} \mathrm{O}_{4}$ core and linker groups). This mechanism can be explained by the coordination chemistry, and thus Pt will be coated on the surface of APTES-coated $\mathrm{Fe}_{3} \mathrm{O}_{4}$ NPs by a reducing agent and the ultrasonic frequency acts as a catalyst, which causes a chemical reaction to accelerate the speed of Pt deposition onto the APTES-coated $\mathrm{Fe}_{3} \mathrm{O}_{4}$ nanoparticle surface. The nonmagnetic Pt NPs will be removed by magnetic separation (step 3). Clearly, the ultrasound cavitation is beneficial to obtain a uniform medium, eliminate the odds of local conditions, increase the reaction speed and form a new phase. Moreover, this method has a shear effect on agglomeration, which is necessary to prepare highly monodisperse NPs.

\subsection{Characterization of $\mathrm{Fe}_{3} \mathrm{O}_{4} /$ Pt nanoparticles}

Figure 2 shows the transmission electron microscopy images and size distributions between the coated and uncoated $\mathrm{Fe}_{3} \mathrm{O}_{4}$ NPs. From the image of Fig. 2(a), a representative TEM image of unmodified $\mathrm{Fe}_{3} \mathrm{O}_{4}$ NPs with diameters ranging from $c a$.
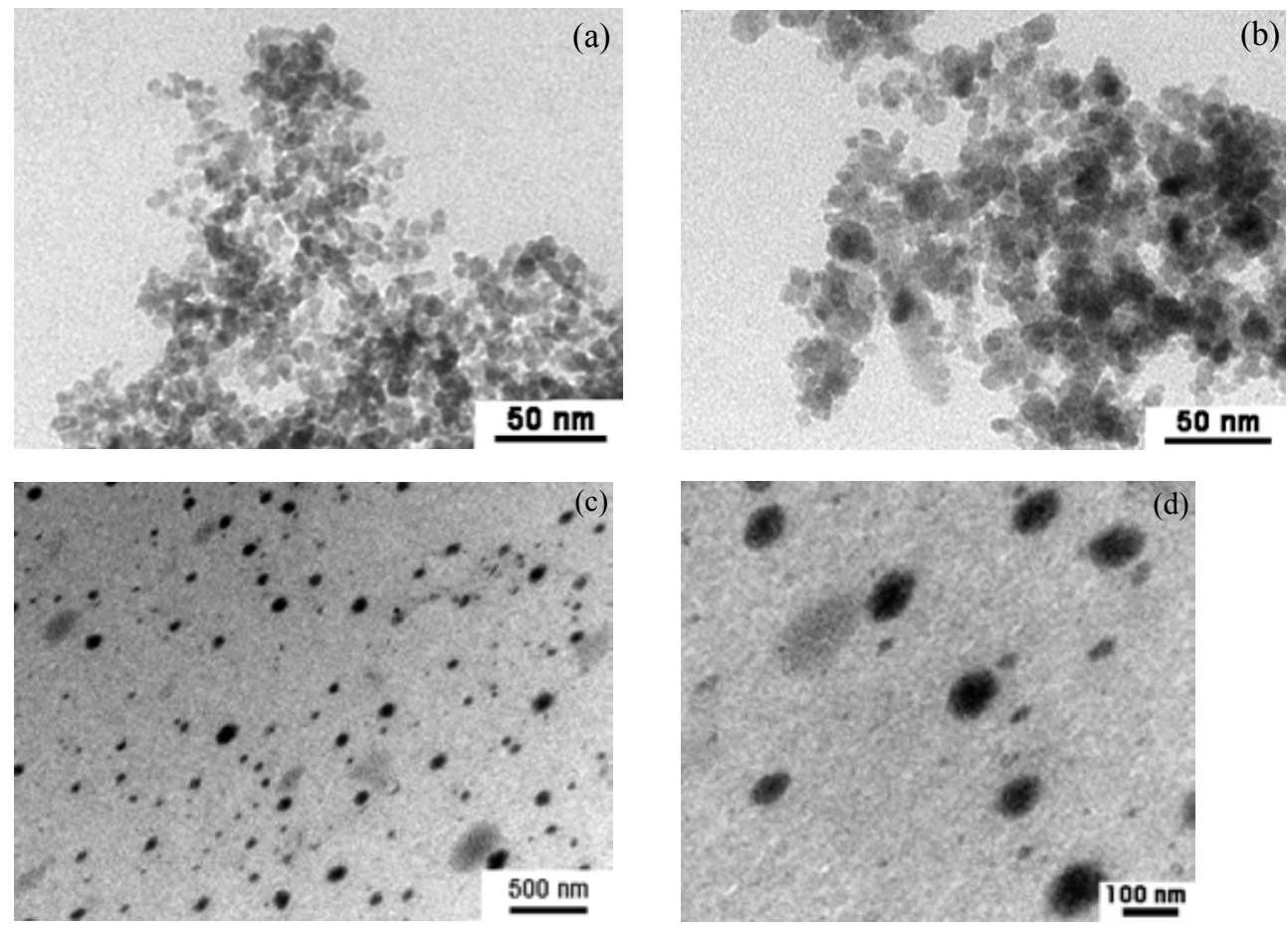

Fig. 2. TEM images of $\mathrm{Fe}_{3} \mathrm{O}_{4}$ (a), APTES- $\mathrm{Fe}_{3} \mathrm{O}_{4}$ (b), and $\mathrm{Fe}_{3} \mathrm{O}_{4} / \mathrm{Pt}(\mathrm{c}, \mathrm{d})$ dispersed in ethanol solution. 
8 to $15 \mathrm{~nm}$ can be seen. The particles display monodispersity and exist in isolation. Figure 2(b) shows TEM images indicating that the average diameter of amino-coated $\mathrm{Fe}_{3} \mathrm{O}_{4}$ NPs was increased to $c a .25 \mathrm{~nm}$ and displays some increase owing to the mild agglomeration of neighboring particles. Figure 2(c) shows a representative TEM image of platinum $(\mathrm{Pt})$-coated $\mathrm{Fe}_{3} \mathrm{O}_{4} \mathrm{NPs}$, and the local amplification image of Fig. 2(c) is shown in the image of Fig. 2(d), which indicates that the average diameter of Ptmodified $\mathrm{Fe}_{3} \mathrm{O}_{4} \mathrm{NPs}$ was increased to approximately $50 \mathrm{~nm}$ and the particles display high monodispersity in size and are well isolated. From the TEM images, it is found that the average particle diameter is increased, but the surface morphology is changed slightly. All the morphologies appear spheroid by comparing images among the $\mathrm{Fe}_{3} \mathrm{O}_{4} \mathrm{NPs}$, the amino-coated $\mathrm{Fe}_{3} \mathrm{O}_{4}$ NPs and the platinum-coated $\mathrm{Fe}_{3} \mathrm{O}_{4}$ NPs. The results suggest that the larger average particle diameter estimated from the image in Fig. 2(c) was caused by the agglomeration of particles and platinum deposition. These findings supported the formation of composite $\mathrm{Fe}_{3} \mathrm{O}_{4} / \mathrm{Pt}$ nanoparticles.

A close inspection of the nanocomposites by using HRTEM revealed that platinum indeed incorporated on the surface of iron oxide. Figure 3 shows the HRTEM images of $\mathrm{Fe}_{3} \mathrm{O}_{4} / \mathrm{Pt}$ nanoparticles. The image of $\mathrm{Fe}_{3} \mathrm{O}_{4} / \mathrm{Pt}$ nanoparticles (Fig. 3(b)) clearly shows
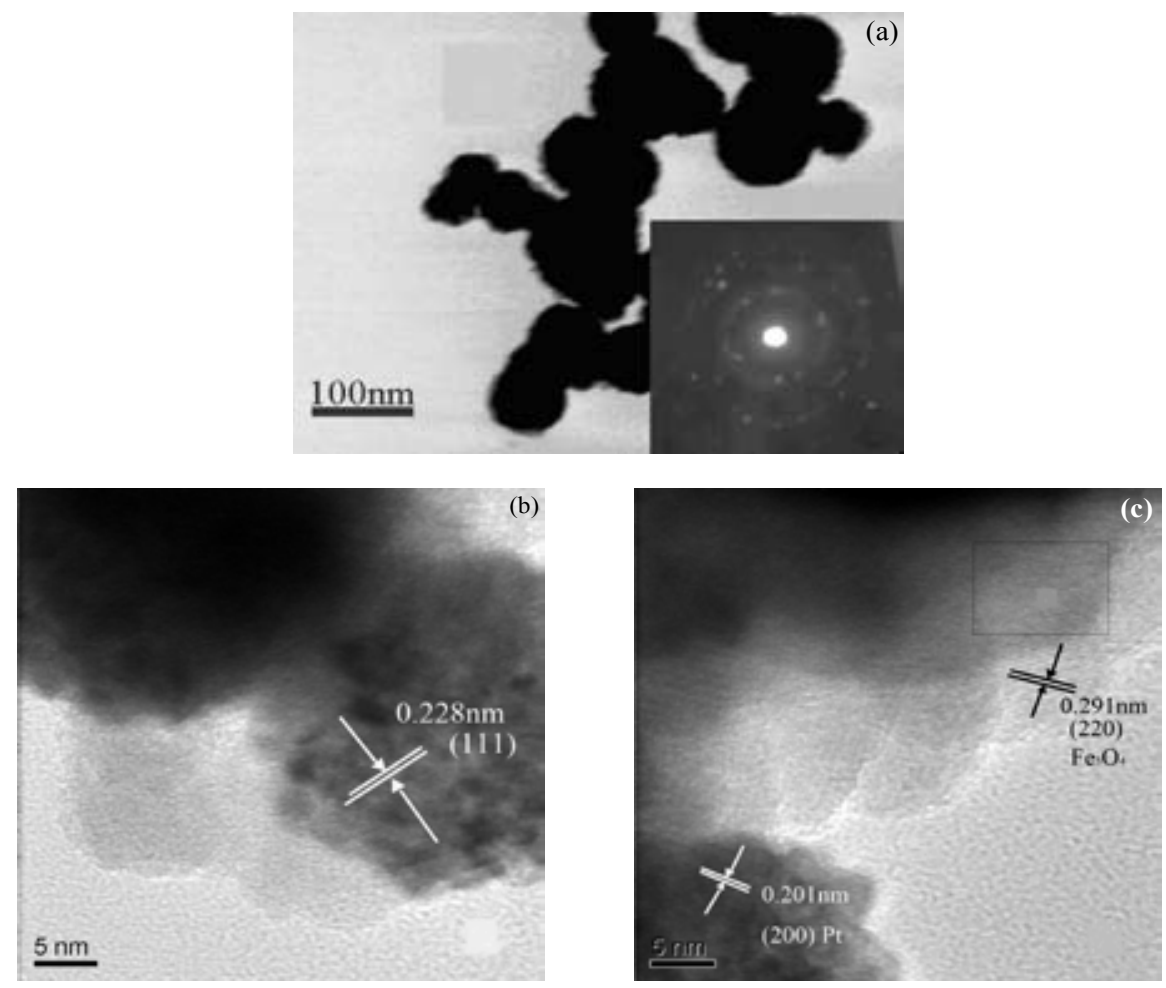

Fig. 3. HRTEM images of $\mathrm{Fe}_{3} \mathrm{O}_{4} / \mathrm{Pt}$ nanoparticles. 
that the interfringe spacing was $0.228 \mathrm{~nm}$, corresponding to the interplanar distance of the 111 crystal plane of platinum metals (01-087-0640, JCPDS-ICDD), which suggests the existence of $\mathrm{Fe}_{3} \mathrm{O}_{4} / \mathrm{Pt}$ nanoparticles. In comparison, HRTEM analysis of the edges of $\mathrm{Fe}_{3} \mathrm{O}_{4} / \mathrm{Pt}$ nanoparticles shows interfringe spacing of $0.291 \mathrm{~nm}$ corresponding to the (220) plane of $\mathrm{Fe}_{3} \mathrm{O}_{4}$ (Fig. 3(c)), further confirming the $\mathrm{Fe}_{3} \mathrm{O}_{4}$ core coexistence with $\mathrm{Pt}$ coating. However, the interfringe of the core region $\mathrm{A}$ of the $\mathrm{Fe}_{3} \mathrm{O}_{4} / \mathrm{Pt}$ nanoparticles (shown in Fig. 3(c)) is difficult to distinguish, owing to the overlapping of fringes between $\mathrm{Fe}_{3} \mathrm{O}_{4}$ and Pt. This may be because the $\mathrm{Fe}_{3} \mathrm{O}_{4}$ nanoparticles were completely encapsulated by the Pt shell.

To confirm the composition of the NPs, X-ray energy dispersive spectroscopy (EDS) spectra were obtained for composition analysis in situ. The result is shown in Fig. 4. EDS indicated the presence of $\mathrm{Fe}, \mathrm{O}$, and $\mathrm{C}$. The presence of a carbon contamination peak comes from the conductive adhesive of the sample substrate. After amino coating and platinum coating, the spectrum shows the presence of N, Si, and Pt. The oxygen peak is also obviously strengthened. The EDS spectrum demonstrated the presence of both Fe and Pt elements in the modified NPs, confirming that the surfaces of magnetite NPs are successfully modified by platinum coating.

To further prove the composition of the NPs, the composites were analyzed by XPS, and the results are plotted in Fig. 5. Figure 5(a) shows the spectral scan of $\mathrm{Fe}_{3} \mathrm{O}_{4} / \mathrm{Pt}$ nanoparticles. There was plenty of carbon existing in the composite nanoparticles, which may result from carbon contamination. From the partial enlarged detail of $\mathrm{Fe}_{2 \mathrm{p}}$, the value of $\mathrm{Fe}_{2 \mathrm{p}}$ appeared at $\mathrm{Fe}_{2 \mathrm{p}}=710.7 \mathrm{eV}$, which was in agreement with the typical value of $\mathrm{Fe}_{2 \mathrm{p}}(710.4 \mathrm{eV})$ in $\mathrm{Fe}_{3} \mathrm{O}_{4}$ species. Figure 5(b) shows the wide-scan XPS spectra for the APTES-coated $\mathrm{Fe}_{3} \mathrm{O}_{4}$ NPs before and after the reaction of platinum modification. The $\mathrm{Cl}$ (2s) and $\mathrm{Cl}(2 \mathrm{p})$ signals were observed in the sample before the reaction, and subsequent

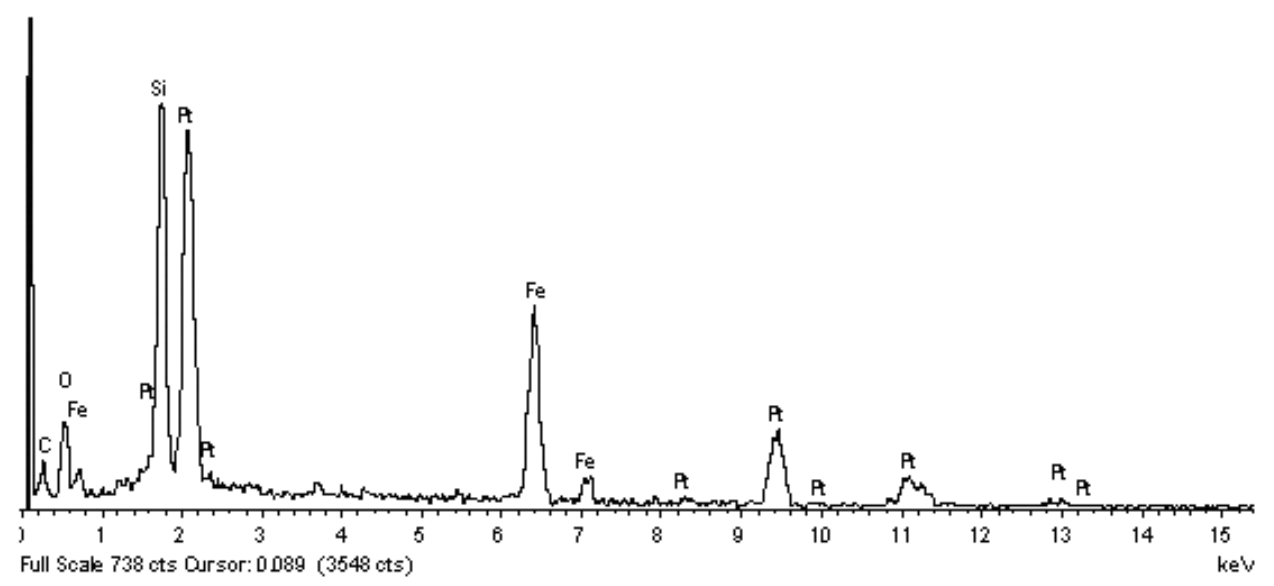

Fig. 4. In situ EDS spectrum of $\mathrm{Fe}_{3} \mathrm{O}_{4} / \mathrm{Pt}$ NPs with identification of the observed peaks. 

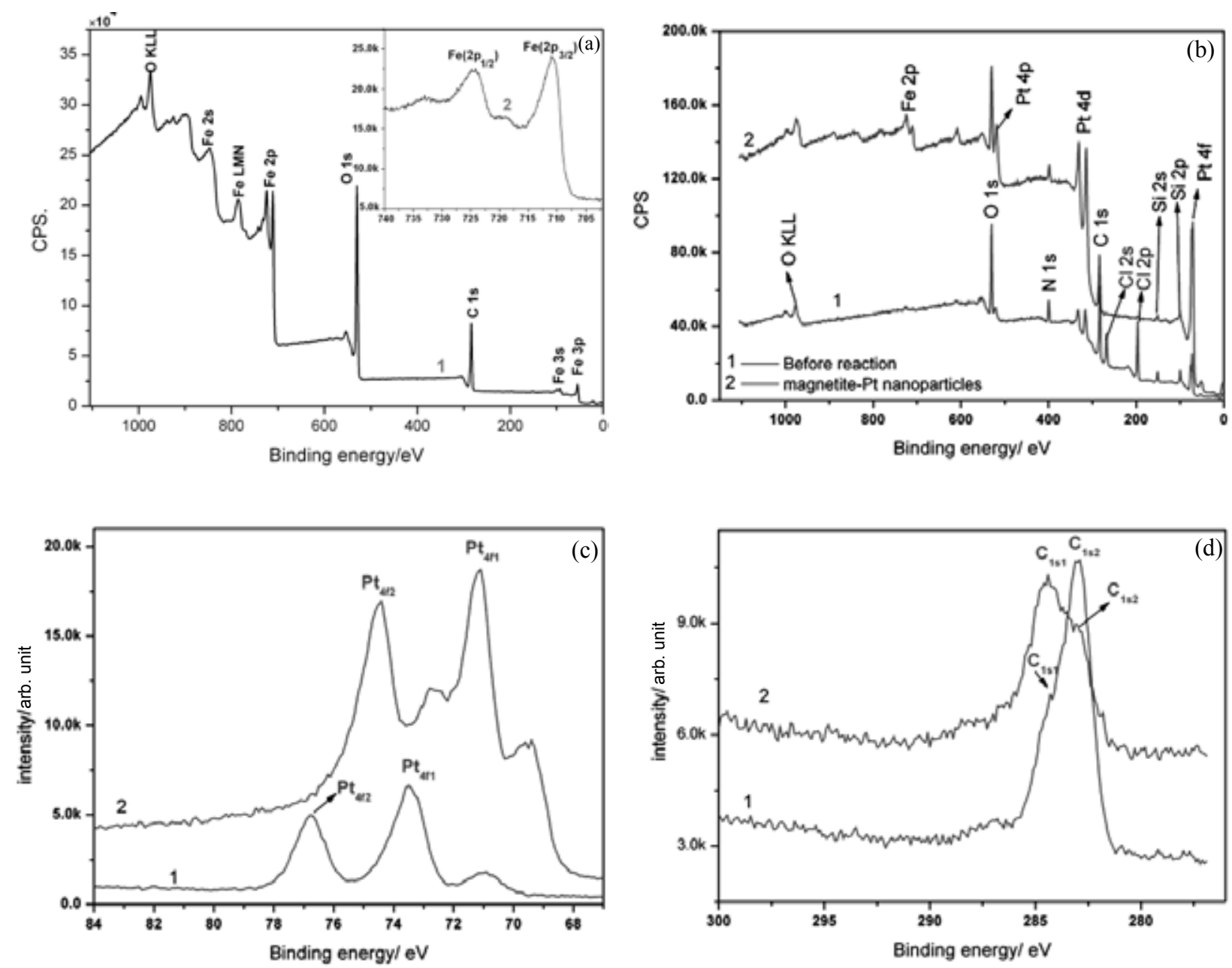

Fig. 5. Samples of $\mathrm{Fe}_{3} \mathrm{O}_{4}$ before Pt capping (1) and Pt-coated magnetite (2): XPS spectra with the $\mathrm{Fe}(2 \mathrm{p})$ region magnified on the top-right corner (a), APTES-modified magnetite nanoparticles before and after Pt-coating reaction (b), regions for $\mathrm{Pt}_{4 \mathrm{f}}(\mathrm{c})$, and $\mathrm{C} 1 \mathrm{~s}(\mathrm{~d})$ comparison.

signals of the $\mathrm{Cl}(2 \mathrm{~s})$ and $\mathrm{Cl}(2 \mathrm{p})$ disappeared in the sample after the ultrasonic platinum reduction reaction. Figure 5(c) shows the regions for $\mathrm{Pt}(4 \mathrm{f})$ comparison. The peak after the reaction of $\mathrm{Pt}$ coating that appeared at $\mathrm{Pt}_{4 \mathrm{f1}}=71.12 \mathrm{eV}, \mathrm{Pt}_{4 \mathrm{f2}}=74.42 \mathrm{eV}$ can be assigned to $\mathrm{Pt}^{0}\left(\mathrm{Pt}_{4 \mathrm{f1}}=71.0 \mathrm{eV}, \mathrm{Pt}_{4 \mathrm{f2}}=74.5 \mathrm{eV}\right)$, but before the reaction, it was at $\mathrm{Pt}_{4 \mathrm{fl}}=73.49 \mathrm{eV}$, $\mathrm{Pt}_{4 \mathrm{f} 2}=76.76 \mathrm{eV}$, apparently arising from Pt (IV) species ([PtCl6 $]^{2-}$ ions). In addition, many studies demonstrated that if particles possessed a core and shell system, the core element would be screened by the shell element, and thus, the compositions in the shell layer become gradually more dominant; the same element intensity ratio of the shell to core species would gradually increase. Figure 5(d) shows the regions for carbon 
comparison. Therefore, corresponding $\mathrm{C}$ signal changes were discerned. The XPS result indicates that the $\mathrm{Fe}_{3} \mathrm{O}_{4}$ core has been coated in a platinum shell format, thus greatly reducing the intensity signals of the element inside.

\subsection{Optical properties}

It is known that nanometer-sized metal clusters usually exhibit unique optical properties with their specific absorption and scattering.(31) Colloidal dispersions of metals exhibit absorption bands or broad regions of absorption in the ultraviolet-visible range. These are due to the excitation of plasma resonances or interband transitions that are a characteristic property of the metallic nature of the particles. ${ }^{(32)}$ Figure 6 shows the absorption spectra of various samples during the platinum coating. It can be seen from Fig. 6 that the hexacholoroplatinum acid hydrate has a strong absorption in the UV region at the peaks of approximately $340 \mathrm{~nm}$, implying optical adsorption characterics of a coordinated complex of $[\mathrm{PtCl} 6]^{2-}$ ions. With further sonochemical reduction, the above peaks started disappearing, suggesting that $[\mathrm{PtCl} 6]^{2-}$ ions were reduced gradually. After the platinum coating, an obvious absorption in the ultraviolet region at the wavelength of $300 \mathrm{~nm}$ and a weak absorption in the visible region are observed, which are due to ligand-to-metal charge transfer transitions of Pt ions. A weak tail stretching across 400-800 nm was finally observed, which is characteristic of Pt nanoparticles. At this stage, the color of the solution was transparent and dark brown.

By comparing ultraviolet-visible absorption data, it can be seen that the absorption in the ultraviolet region of amino-coated $\mathrm{Fe}_{3} \mathrm{O}_{4} \mathrm{NPs}$ was weaker than that in $\mathrm{Fe}_{3} \mathrm{O}_{4} \mathrm{NPs}$, and the absorption of platinum-coated $\mathrm{Fe}_{3} \mathrm{O}_{4}$ NPs was weaker than that of the amino-

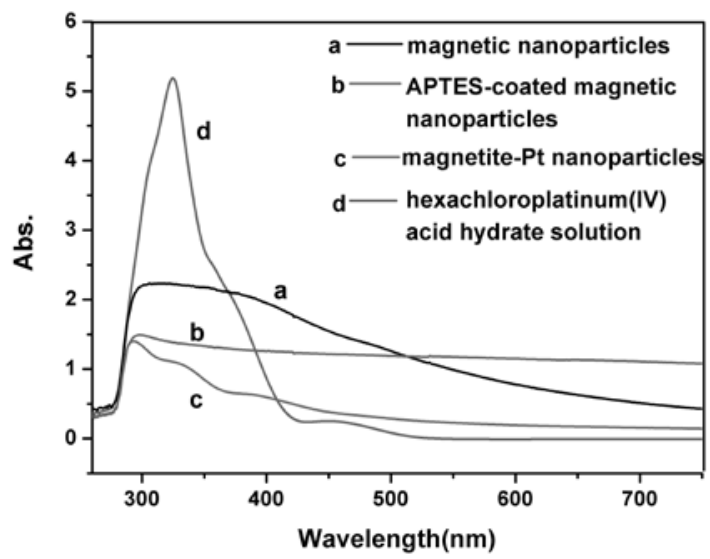

Fig. 6. UV-Vis spectra comparison of $\mathrm{Fe}_{3} \mathrm{O}_{4}$ nanoparticles (a), APTES-coated $\mathrm{Fe}_{3} \mathrm{O}_{4}$ nanoparticles (b), $\mathrm{Fe}_{3} \mathrm{O}_{4} / \mathrm{Pt}$ composite nanoparticles (c), and the hexacholoroplatinum acid hydrate solution (d). 
coated $\mathrm{Fe}_{3} \mathrm{O}_{4}$ NPs. This was mostly due to the effect of APTES and platinum surface modification, which can improve and strengthen the ultraviolet absorption, but weak absorption remains in the visible region. The visible absorptions for both have no difference in terms of peaks, but distinguished from metal-coated magnetic NPs, such as gold-modified NPs, which show a strong absorption peak as a result of plasmon resonance (SP). ${ }^{(33)}$ This also proves that the platinum was coated on the magnetite and thus the coated nanoparticles can enhance its optical properties to some extent.

\subsection{Magnetic properties}

The nanoparticle hysteresis loops measured (at $T=300 \mathrm{~K}$ and $T=5 \mathrm{~K}$ ) in a field of 100 Oe on a SQUID magnetometer are shown in Fig. 7, which depicts the hysteresis loops of $\mathrm{Fe}_{3} \mathrm{O}_{4}$ and Pt-coated $\mathrm{Fe}_{3} \mathrm{O}_{4}$ NPs (a) and magnetic isolation photograph of Pt and Ptcoated $\mathrm{Fe}_{3} \mathrm{O}_{4} \mathrm{NPs}(\mathrm{b})$. Determination numbers from the SQUID magnetometry reveal that overlaying the $\mathrm{Fe}_{3} \mathrm{O}_{4}$ NP surface with a shell of Pt causes a decrease in magnetic behavior. The saturation magnetization $\left(M_{\mathrm{s}}\right)$ of bare NPs was found to be $63.7 \mathrm{emu} \mathrm{g}^{-1}$ at $300 \mathrm{~K}$ and $70.3 \mathrm{emu} \mathrm{g}^{-1}$ at $5 \mathrm{~K}$. In contrast, the $M_{\mathrm{s}}$ of Pt-coated $\mathrm{Fe}_{3} \mathrm{O}_{4} \mathrm{NPs}$ was found to be $23.8 \mathrm{emu} \mathrm{g}^{-1}$ at $300 \mathrm{~K}$ and $27.3 \mathrm{emu} \mathrm{g}^{-1}$ at $5 \mathrm{~K}$. Obviously, the $M_{\mathrm{s}}$ at $300 \mathrm{~K}$ is lower than that at $5 \mathrm{~K}$, which agrees with the formula that increasing the temperature would cause the $M_{\mathrm{s}}$ to decrease. The above interpretation in terms of a surface anisotropy, as a result of the interaction with outer layers like the platinum coating, indicates that the surface anisotropy probably also affects the moment of the inner $\mathrm{Fe}_{3} \mathrm{O}_{4}$ nanoparticles via the exchange interaction with the platinum coating layer at the surface. ${ }^{(34)}$ Thus, the $M_{\mathrm{s}}$ of $\mathrm{Fe}_{3} \mathrm{O}_{4}$ NPs decreased along with the immobilization of the Pt shell on their surface.

The noble metal nanoparticles with so-called surface effect at nanoscale dimensions
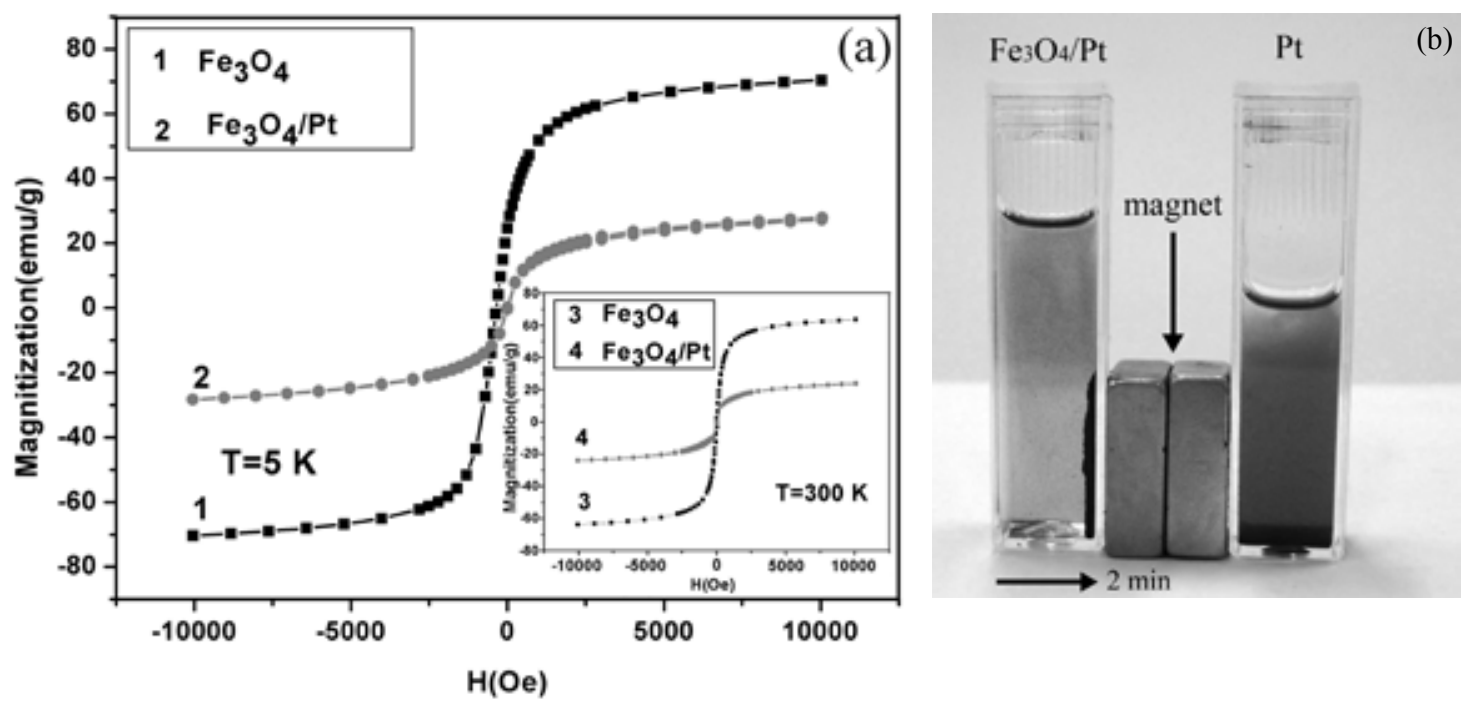

Fig. 7. Hysteresis loops of $\mathrm{Fe}_{3} \mathrm{O}_{4}$ and $\mathrm{Fe}_{3} \mathrm{O}_{4} / \mathrm{Pt}$ nanoparticles measured at $T=300 \mathrm{~K}$ and $T=5 \mathrm{~K}$ (a) and magnetic isolation photograph of $\mathrm{Fe}_{3} \mathrm{O}_{4} / \mathrm{Pt}$ (left) and $\mathrm{Pt}$ nanoparticles (right) (b). 
are interesting in the catalytic field, although there still remains a major problem relating to the simple and rapid technology availability for catalyst separation and recycling. Figure 7(a) is an image of a simple experimental demonstration, showing that the $\mathrm{Fe}_{3} \mathrm{O}_{4}$ NPs after metal platinum coating still have a good magnetic response, and it can be easily extracted by magnetic separation. Meanwhile, the demonstration simply proves that the metallized platinum coating does not substantially affect the bulk magnetism of magnetite, although it decreases the $M_{\mathrm{s}}$ of these nanocomposites.

\section{Conclusions}

In summary, nanocomposites of platinum-coated magnetite nanoparticles $\left(\mathrm{Fe}_{3} \mathrm{O}_{4} @\right.$ Pt NPs) are prepared via surface reduction of platinum coating on APTES-functionalized magnetite nanoparticles under ultrasound application in aqueous solution media. The monodisperse $\mathrm{Fe}_{3} \mathrm{O}_{4} @$ Pt NPs of $c a .50$ nm were characterized by TEM, HRTEM, EDS, XPS, UV-Vis, and SQUID magnetometry, and these nanocomposites preserved not only the inner magnetism of the magnetite core but also the catalytic noble metal of platinum on the surface shell. The interesting magnetic responsive phenomena of the $\mathrm{Fe}_{3} \mathrm{O}_{4} @$ Pt NPs will potentially expand its application in a magnetic catalytic field. Such a onepot-synthesis method without any surfactant was proven to be simple, inexpensive and versatile. The $\mathrm{Fe}_{3} \mathrm{O}_{4} @$ Pt NPs offer a useful reference and thus can be extended to other metal/metal oxide compositions towards magnetosensitive catalyst fabrication.

\section{Acknowledgements}

The authors gratefully acknowledge financial support from the Natural Science Foundation of China (Nos. 61102031, 61001053), University Creative Group Project of Hunan Education Department, Scientific Research Fund of Hunan Provincial Education Department (No. 08A013), Natural Science Foundation of Hunan Province (Nos. 11JJ6038, 11JJ5042) and Postgraduates Scientific Innovation Fund of Hunan Province (CX2010B398).

\section{References}

1 R. P. Andres, J. D. Bielefeld, J. I. Henderson, D. B. Janes, V. R. Kolagunta, C. P. Kubiak, W. J. Mahoney and R. G. Osifchin: Science 273 (1996) 1690.

2 H. Hirai, H. Wakabayashi and M. Komiyama: Chem. Lett. 12 (1983) 1047.

3 R. S. Sonawane, B. B. Kale and M. K. Dongare: Mater. Chem. Phys. 85 (2004) 52.

4 J. S. Cheng, Y. Wu, Z. H. Xu, B. Hu, J. M. Bai, J. P. Wang and J. Shen: Appl. Phys. Lett. 90 (2007) 23.

5 T. Philippe, D. Robert, C. Bernard, C. Christophe, R. Séverine and L. Lamy: Appl. Catalysis B: Environmental 93 (2009) 280.

6 T. Vinay, J. K. Jiang and S. Virendra: Appl. Catalysis A: General. 345 (2008) 241.

7 J. A. A. J. Perenboom, P. Wyder and F. Meier: Phys. Rep. 78 (1981) 173.

8 J. D. G. Durán, J. L. Arias, V. Gallardo and A. V. Delgado: J. Pharm. Sci. 97 (2008) 2948.

9 D. Astruc, F. Lu and J. R. Aranzaes: Angew. Chem. Int. Ed. 44 (2005) 7852. 
10 G. Schmid: Nanoscale Materials in Chemistry, ed. K. J. Klabunde (Wiley-Interscience, New York, 2001) p. 15.

11 J. H. Fendler and Y. Tian: Nanoparticles and Nanostructured Films: Preparation, Characterisation and Applications in Nanoparticles and Nanostructured Films, ed. J. H. Fendler (Wiley-VCH, Weinheim, Germany, 1998) p. 429.

12 M. M. Alvarez, J. T. Khoury, T. G. Schaaff, M. N. Shafigullin, I. Vezmar and R. L. Whetten: J. Phys. Chem. B 101 (1997) 3706.

13 B. M. Quinn, P. Liljeroth, V. Ruiz, T. Laaksonen and K. Kontturi: J. Am. Chem. Soc. 125 (2003) 6644.

14 S. Kidambi and M. L. Bruening: Chem. Mater. 17 (2005) 301.

15 Y. Niu, L. K. Yeung and R. M. Crooks: J. Am. Chem. Soc. 123 (2001) 6840.

16 P. V. Kamat: J. Phys. Chem. C. 111 (2007) 2834.

17 J. H. Park, Y. T. Lim and O. O. Park: Chem. Mater. 16 (2004) 688.

18 K. Yakushiji, F. Ernult, H. Imamura, K. Yamane, S. Mitani, K. Takanashi, S. Takahashi, S. Maekawa and H. Fujimori: Nat. Mater. 4 (2005) 57.

19 J. S. Ye, A. Ottova, H. T. Tien and F. S. Sheu: Bioelectrochem. 59 (2003) 65.

20 E. E. Carperter, S. Calvin and R. M. Stroud: Chem. Mater. 15 (2003) 3245.

21 P. Gong, H. Li and X. He: Nanotechnol. 18 (2007) 5604.

22 I. Y. Goon, M. H. Lai Leo, M. Lim, P. Munroe, J. J. Gooding and R. Amal: Chem. Mater. 21 (2009) 673.

23 M. Leonowicz, M. Wozniak, Y. M. Shulga, V. E. Muradyan, Z. Liu, H. A. Davies and W. Kaszuwara: Mater. Lett. 60 (2006) 442.

24 J. Qiu, H. Peng and R. Liang: Electrochem. Commun. 9 (2007) 2734.

25 W. Wang, J. Luo and Q. Fan: J. Phys. Chem. B. 109 (2005) 21593.

26 Y. Wu, J. Guo, W. Yang, C. Wang and S. Fu: Polymer. 47 (2006) 5287.

27 S. Wang and Y. Tan: Anal. Bioanal. Chem. 387 (2007) 703.

28 Q. Li, H. Li, V. G. Pol, I. Bruckental, Y. Koltypin, J. Calderon-Moreno and I. Nowik: New J. Chem. 27 (2003) 1194.

29 T. Gao, Q. Li and T. Wang: Chem. Mater. 17 (2005) 887.

30 W. Wu, Q. He, H. Chen, J. Tang and L. Nie: Nanotechnol. 18 (2007) 145609.

31 J. A. Creighton and D. G. Eadon: Faraday Trans: Chem. Soc. 87 (1991) 3881.

32 J. Wang, W. Wu and D. Feng: Introduction of Electronic Energy Spectrum (XPS/XAES/UPS) (National Defence Industry Press, Peking, 1992) p. 519.

33 K. J. Brown, D. G. Walter and M. Natan: Chem. Mater. 11 (2000) 306.

34 P. M. Paulus, H. Bönnemann, A. M. van der Kraan, F. Luis, J. Sinzig and L. J. de Jongh: Euro. Phys. 9 (1999) 501.

35 C. K. Tsung, J. N. Kuhn, W. Huang, C. Aliaga, L. Hung, G. A. Somorjai and P. Yang: J. Am. Chem. Soc. 131 (2009) 5816.

36 S. H. Joo, J. Y. Park, C. K. Tsung, P. Yang and G. A. Somorjai: Nat. Mater. 8 (2009) 126. 